\title{
Caring about symptoms in person-centred care
}

\author{
Eva Brink ${ }^{1,2,3}$, Carola Skott ${ }^{2}$ \\ ${ }^{1}$ Department of Nursing, Health and Culture, University West, Trollhättan, Sweden \\ ${ }^{2}$ Institute of Health and Care Sciences, University of Gothenburg, Gothenburg, Sweden \\ ${ }^{3}$ Centre for Person-Centred Care (GPCC), University of Gothenburg, Gothenburg, Sweden \\ Email: eva.brink@hv.se
}

Received 10 October 2013; revised 12 November 2013; accepted 26 November 2013

Copyright (C) 2013 Eva Brink, Carola Skott. This is an open access article distributed under the Creative Commons Attribution License, which permits unrestricted use, distribution, and reproduction in any medium, provided the original work is properly cited.

\begin{abstract}
In the present article, we emphasize the symptom experience perspective in person-centred care and discuss barriers to implementation of this approach. There are obstacles to overcome: the diversity of understandings of symptoms in clinical settings, the current biomedical discourse and the incompleteness of symptom research. Since the 19th century, the biomedical perspective has been powerful in conceptualizing symptoms in terms of pathology and diagnosis. Many diagnoses conjure up preconceived notions about the persons receiving them. This perspective may influence person-centred care negatively. Yet symptoms often mean something beyond the diagnosis. Recognizing this discrepancy, it is crucial that we consider a perspective that starts from each person's symptom experience, thus complementing the biomedical perspective. Using the notion caring about symptoms, we advocate a person-centred approach that includes a symptom experience perspective. This requires health-care professionals to be skilled in listening to patient narratives and acquire knowledge about how symptom experiences can be individually expressed and interpreted. Listening to symptom experiences may give insights into the personal meaning of illness as well as information about bodily and social restrictions caused by symptom distress. In this way, caring about symptoms will improve the prerequisites for establishing person-centred care planning.
\end{abstract}

Keywords: Biomedical Perspective; Nursing Practice; Patient-Centred Care; Symptom Experience

\section{INTRODUCTION}

Symptoms are at the core of communication and dialogue whenever patients and professionals meet in health-care situations. Patients' descriptions of their illness and distress are significant to professional understanding of their suffering. Sensations and perceptions have to be translated into and expressed in words. However, in a clinical setting, subjective symptom experience is filtered through a professional interpretation aimed at a specific diagnosis. As long as health-care professionals put disease manifestations in the foreground (a disease-centred approach) and patients' own symptom experiences in the background, we must push for care models that ensure a more individualized perspective on symptoms and symptom management. We need strategies for integrating experiential knowledge into clinical practice.

Promoting a person-centred approach in which the patient's experience comes first would seem to be self-evident. Such an approach, aimed at humanizing the healthcare system through new health-care models, was presented early on [1] and has been pursued internationally during recent decades under various designations: person-centredness [2,3], patient-centered care [4], personcentred nursing [5], and person-centred care [6]. Although frequently used in the literature, there are several interpretations of the concept person-centered care [7] and person-centredness [8]. However, the concept basically asserts that the patient is a person and that care provision efforts should be based on personal illness experiences.

In a person-centred approach to care, each person should be seen as completely unique, meaning that different persons with similar symptom experiences should not automatically be treated or supported in the same way. This leads to dilemmas in everyday health care, as the negotiation of symptoms, embedded in a singular search for a diagnosis, constitutes the central concern of most health-care encounters. In this paper, we discuss a person-centred care approach and explore the barriers to implementation of a symptom experience perspective in person-centred care. 


\section{BARRIERS TO CARING ABOUT SYMPTOMS}

\subsection{Diversity of Understandings of Symptoms in Clinical Settings}

In the context of scientific medicine, the term symptom refers to the subjective experience of illness, not directly observable, while signs are alterations that can be detected through the observer's senses and denoted by objective biomedical disease markers, e.g. visible physical marks and laboratory findings $[9,10]$. Sometimes relationships between signs and symptoms can be identified, but such associations are more often lacking [11]. The predominant biomedical approach is to conceptualize symptoms in terms of pathology [9,12]. The meaning of the term therefore becomes blurred. Note that use of the term symptom as an all-embracing marker of disease may still be widespread in everyday health-care practice.

During the 1970s and 1980s, attention was turned to symptoms as subjective illness experiences [13,14]. However, subjective measures of disease and treatment impact are still viewed with scepticism in biomedical practice [15]. Therefore, health-care professionals are expected to translate the patient's complaints into that which is considered authentic: biological signs of disease. This may influence person-centred care negatively, as many diagnoses conjure up preconceived notions of the persons receiving them [16]. There is a tendency to medicalize life experiences and to translate social suffering into bodily dysfunctions. In this way, suffering is turned into something medically and socially legitimate, while the personal experience is left aside.

At times unreflective use of biomedical language threatens to undermine the patient's confidence [17]. By providing explanations that question the reality or legitimacy of symptoms, professionals risk losing patients' confidence [18]. If health-care professionals cannot define, experience or measure patients' symptoms, they will under-report and thereby under-treat these symptoms [19]. Inattention to symptom experiences may have negative consequences and in some cases constitute an essential part of the patient's suffering.

\subsection{Power of Interpretation-Biomedicine}

Since the 19th-century development of pathological anatomy, physicians have regarded disease as a localized process mapped onto specific, malfunctioning internal organs [9]. Biomedical discourse is specific and powerful. It differs from other forms of discourse by its insistence on materialism as the basis of knowledge, and because of its requirement that single causal chains must be used to specify pathogenesis [20]. Biomedicine also presupposes that what is experienced can be fully expressed in words, and as a sign of efficacy it is even preferable that symp- toms be considered as one-word expressions. Diagnostic techniques and procedures may be trained and practiced using symptom handbooks, manuals and "symptom sorters" [21,22].

When patients in a clinical setting express atypical or unexpected symptoms, they are sometimes met with incomprehension. Many patients therefore struggle to translate their experience into a more legitimate language and use a medical idiom to explain their symptoms. This impoverishes their language, such as when common oneword symptoms like fatigue, pain, anxiety or dizziness conceal diverse and varying experiences. Medical authority limits and restricts the range of acceptable meanings so as to adhere to an established diagnostic process [23].

Patients learn to communicate in a way that differs significantly from their talk in everyday life. They also learn what symptoms they had better be silent about. When symptoms indicate the existence of something other than a legitimate disease, there is a risk of creating a growing distance between patients and professionals. When symptoms are not recognized by applying biomedical knowledge, we speak of medically unexplained symptoms (MUS). These kinds of symptoms comprise half the volume of emergency health care consultations and represent the most common diagnosis in some specialties [24]. Pathology-based approaches to symptoms are increasingly being found to be both scientifically and clinically inadequate. An alternative approach is to regard symptoms as personal experience and an expression of a combination of biological, psychological and social factors. Further, in a person-centred care context, such an approach confirms the trust between the patient and the health-care professional.

\subsection{Incompleteness of Symptom Research}

Research is needed that is grounded on subjective symptom experiences including the meaning of symptoms and how to perceive, express, live with and handle them. In fact, there is a great deal of existing research in this area, but some agreement regarding concept definitions would be desirable. To promote the development of a broad knowledge base, it is of vital importance that different methodologies be applied. A variety of symptom concepts are found in this research area, a few of which will be mentioned here.

The diversity of concepts surrounding symptoms has to be dealt with, because some of the concepts are vague or overlapping in their definitions. For example, symptom experience has been described as including both symptom occurrence (quality, frequency and duration) and symptom distress [10]. According to Dodd et al. [25], symptom experience is a dynamic process, which involves symptom perception, evaluation of its meaning 
and response to a symptom. This model could be criticized for not specifying the directionality between multidimensional indicators and symptom status. Armstrong [26] included the following terms in the symptom experience concept: perception of the frequency, intensity, distress, meaning of symptoms as they are produced and expressed, and personal consequences.

Closely connected to symptom experience is the concept symptom perception, which covers the belief a person has about what a particular symptom means cognitively and emotionally. Bodily cues, psychological factors, and situational factors as well as knowledge and earlier experiences are said to interact in the symptom perception process $[27,28]$.

Much research remains to be done in this vital research area to develop a significant and coherent knowledge base for considering symptoms in clinical praxis. A framework for handling the diversity of the concept should be helpful. It would require more theoretically rooted empirical studies for clarification of concepts and exploration of dynamic interaction effects.

\section{A SYMPTOM EXPERIENCE PERSPECTIVE IN PERSON-CENTRED CARE}

The position of symptoms in person-centred care is paramount. Person-centred care planning starts with patient narratives of unique illness experiences. The health-care professional seeks to understand these experiences through the patient narratives, and should try to understand what the symptom or various symptoms mean to the patient. Symptoms often represent a call for help, reflecting not only physical aspects but also anxiety, depression, fear and expectations. The person's subjective experience of illness has both cultural significance and a personal meaning regarding changes in health. This experience has to be conveyed and interpreted. Narrative communication, with its metaphors and foundation in human life, provides the basis for expressing vulnerability and fears [29-31]. Important issues of concern in person-centred care deal with the distress caused by symptoms as well as the desire for symptom relief. Eldh et al. [32] showed that patient participation was optimized when the patient was regarded as a person and when the patient's symptom narratives were recognized by staff. In patients hospitalized for symptoms of worsening chronic heart failure, implementation of a person-centred approach shortened the hospital stay. Moreover, this effect was achieved without negative patient outcomes [33]. Furthermore, person-centred care is positively correlated with a constructive change of perspective [34] and job satisfaction [35].

The obstacles to implementation of person-centred care including a symptom experience perspective are manifold and multileveled. There is certainly no one-way road leading from pathology to perception and then to expression and verbalization of symptoms, and we still have inadequate knowledge of how words for symptoms relate to experience. The word is not the experience itself, but the mediation of experience made meaningful. The mystery of how physiological expression is transformed into verbal information is still unsolved. However, the social environment decides what is understood as a relevant symptom and determines the choice of words used to convey that symptom. Symptom interpretation solely aimed at diagnosis and optimal medical treatment of disease may be unsatisfactory. Illness is a subjective experience, and correlations between such an experience and malfunctioning internal organs do not always exist [14]. Symptoms often mean something over and above the diagnosis, which may cause discordance. Bodily impediments, complicated emotions or obstacles to daily activities may be experienced by patients as symptoms, i.e. as actual indications of disease, while professionals may interpret these markers differently [36]. The gap between the biomedical discourse and the patient's experience may create misunderstandings, and therefore, a great deal of caution is needed to prevent communication distress. Recognizing this discrepancy, it would seem crucial to have a perspective that starts from a person's own symptom experience, in this way complementing the biomedical perspective. Corwin et al. [37] pointed out the negative aspects of separating the determinants of a symptom from the phenomena associated with that symptom. Instead, specifying causal sequences and reciprocal relationships between bio-behavioural determinants and phenomena should guide future symptom research. Until we have understood the symptom interpretation process from the individual's perspective and linked it to models of care, our skills in helping patients manage symptoms will be limited.

A shift from a "disease-centred" to a "person-centred" approach cannot just happen on its own. We need a model that provides a clarification of complexity and simultaneously reveals how the caring process fits in. Armstrong [26] argued that, in current studies and theories of symptom evaluation, the meaning of the symptom experience to patients is far from sufficiently considered. In an attempt to present a meaning-centred approach, she suggests using a multilevel model of symptoms (p. 603). This model includes the antecedents to and production, perception, expression and consequences of symptoms. The model also includes the meaning of symptoms and the existential meaning of symptom expression. Such a model might be a step forward in health-care professionals' endeavours to grasp the complexity of patients' symptom experience. However, the model is silent about the most essential step in the care process: the dialogue 
between the patient and the health-care professional, which always occurs in a specific context. This aspect could usefully be added to the model.

Personalized care actions for symptom relief should be developed through dialogue and shared decisions between the patient and the health-care professional, which runs contrary to standardized routine measures. Effective implementation of person-centred care is possible only when management follows through with plans, when care teams have confidence in their ability to meet goals for change, and when change fosters smooth operations in the daily routines of direct care providers [38]. A person-centred integrative diagnosis (PID) of the whole picture of a person's health has been suggested [39]. The aim is not solely to provide a diagnosis, but also to portray the actual illness experiences and support fulfilment of the person's health aspirations and personal needs. Person-centred care relies on a partnership between the patient and the health-care professional, which is initiated by the patient's narrative [40].

There are many obstacles to overcome when implementing person-centred care that involves a symptom experience perspective: the diversity of understandings of symptoms in clinical settings, the current biomedical discourse and the incompleteness of symptom research. Therefore, establishing person-centred care in a healthcare unit requires commitment across the entire organization, that is, a movement towards a shared culture of person-centred care at all levels, not only temporary personal experiences of person-centredness on the part of staff $[41,42]$.

\section{CONCLUSIONS}

Using the notion caring about symptoms, we advocate a person-centred care perspective based on a dialog between the patient and the health-care professional. This requires the development of professional skills that focus on listening to patient narratives that include the life situation and illness experiences and more specific knowledge about how symptom experiences can be individually expressed and interpreted. The health-care professional could strengthen a person's involvement in treatment and care decisions by presenting a humble understanding of personal symptom experiences in addition to diagnostic techniques and procedures. Also, symptom relief strategies have to be considered in a dialogue with the patient. In contemporary health-care contexts, it is of vital importance to explore symptom experiences and symptom relief more fully. By upgrading the status of symptoms as providing valuable insights into a patient's illness experience, health-care professionals may be able to meet the basic criteria for person-centred care.

Listening to symptom experiences may give insights into the personal meaning of illness as well as information about the potential activity and social restrictions caused by symptom distress. In this way, caring about symptoms will improve the prerequisites for establishing person-centred care planning. However, further empirical research and symptom concept analyses are needed that explore symptom experiences more fully.

\section{REFERENCES}

[1] Balint, E. (1969) The possibilities of patient-centred medicine. Royal College of General Practitioners, 17, 269276.

[2] Leplege, A., Gzil, F., Cammelli, M., Lefeve, C., Pachoud, B. and Ville, I. (2007) Person-centredness: Conceptual and historical perspectives. Disability and Rehabilitation, 29, 1555-1565. http://dx.doi.org/10.1080/09638280701618661

[3] Mead, N. and Bower, P. (2000) Patient-centredness: A conceptual framework and review of the empirical literature. Social Science \& Medicine, 51, 1087-1110. http://dx.doi.org/10.1016/S0277-9536(00)00098-8

[4] Stewart, M. (2001) Towards a global definition of patient centred care. BMJ, 322, 444-445. http://dx.doi.org/10.1136/bmj.322.7284.444

[5] McCormack, B. and McCance, T.V. (2006) Development of a framework for person-centred nursing. Journal of Advanced Nursing, 56, 472-479. http://dx.doi.org/10.1111/j.1365-2648.2006.04042.x

[6] Edvardsson, D., Winblad, B. and Sandman P.O. (2008) Person-centred care of people with severe Alzheimer's disease: Current status and ways forward. Lancet Neurology, 7, 362-367. http://dx.doi.org/10.1016/S1474-4422(08)70063-2

[7] Morgan, S.S. and Yoder, L. (2011) A concept analysis of person-centered care. Journal of Holistic Nursing, 30, 615. http://dx.doi.org/10.1177/0898010111412189

[8] Slater, L. (2006) Person-centredness: A concept analysis. Contemporary Nursing, 23, 135-144. http://dx.doi.org/10.5172/conu.2006.23.1.135

[9] Edwards, M. (2008) Symptoms. Lancet, 371, 1157. http://dx.doi.org/10.1016/S0140-6736(08)60510-3

[10] McDaniel, R.W. and Rhodes, V.A. (1995) Symptom experience. Seminars in Oncology Nursing, 11, 232-234. http://dx.doi.org/10.1016/S0749-2081(05)80002-6

[11] Ekman, I., Cleland, J.G., Andersson, B. and Swedberg, K. (2005) Exploring symptoms in chronic heart failure. European Journal of Heart Failure, 7, 699-703. http://dx.doi.org/10.1016/j.ejheart.2005.07.003

[12] Sharpe, M. and Walker, J. (2009) Symptoms: A new approach. Psychiatry, 8, 146-148. http://dx.doi.org/10.1016/j.mppsy.2009.03.016

[13] Kleinman, A., Eisenberg, L. and Good, B. (1978) Culture, illness, and care. Clinical lessons from anthropological and cross-cultural research. Annals of Internal Medicine, 88, 251-258.

http://dx.doi.org/10.7326/0003-4819-88-2-251 
[14] Good, B. and Delveccio-Good, M.J. (1981) The meaning of symptoms: A cultural hermeneutic model for clinical practice. In: Eisenberg, L. and Kleinman A., Eds., The Relevance of Social Science for Medicine, Reidel Publishing Company, Dordrecht, 165-196. http://dx.doi.org/10.1007/978-94-009-8379-3_8

[15] Sim, J. and Madden, S. (2008) Illness experience in fibromyalgia syndrome: A metasynthesis of qualitative studies. Social Science and Medicine, 67, 57-67. http://dx.doi.org/10.1016/j.socscimed.2008.03.003

[16] McElvaney, C. (2011) Client evaluations and summaries: How person-centered planning is tainted by a diagnosis. Intellectual and Development Disabilities, 49, 203-205. http://dx.doi.org/10.1352/1934-9556-49.3.203

[17] Reisfield, G.M. and Wilson, G.R. (2004) Use of metaphor in the discourse on cancer. Journal of Clinical Oncology, 22, 4024-4027. http://dx.doi.org/10.1200/JCO.2004.03.136

[18] Peters, S., Stanley I., Rose, M. and Salmon, P. (1998) Patients with medically unexplained symptoms: Sources of patients' authority and implications for demands on medical care. Social Science Medicine, 46, 559-565. http://dx.doi.org/10.1016/S0277-9536(97)00200-1

[19] Visentin, M., Zanolin, E., Trentin, L., Sartori S. and de Marco, R. (2005) Prevalence and treatment of pain in adults admitted to Italian hospitals. European Journal of Pain, 9, 61-67. http://dx.doi.org/10.1016/j.ejpain.2004.04.004

[20] Kleinman, A. (1995) Writing at the margin: Discourse between anthropology and medicine. University of California Press, Berkeley.

[21] El-Radhi, S.A. (2011) Paediatric symptom sorter. Radcliffe Publishing Ltd., Oxford.

[22] Hopcroft, K. and Forte, V. (2010) Symptom sorter. 4th Edition, Radcliffe Publishing Ltd., Oxford.

[23] Kirmayer, L.J. (1994) Improvisation and authority in illness meaning. Culture, Medicine and Psychiatry, 18, 183214. http://dx.doi.org/10.1007/BF01379449

[24] Nimnuan, C., Hotopf, M. and Wessely, S. (2001) Medically unexplained symptoms: An epidemiological study in seven specialities. Journal of Psychosomatic Research, 51, 361-367. http://dx.doi.org/10.1016/S0022-3999(01)00223-9

[25] Dodd, M., Janson, S., Facione, N., Faucett, J., Froelicher, E.S., Humphreys, J., Lee, K., Miaskowski, C., Puntillo, K., Rankin, S. and Taylor, D. (2001) Advancing the science of symptom management. Journal of Advanced Nursing, 33, 668-676. http://dx.doi.org/10.1046/j.1365-2648.2001.01697.x

[26] Armstrong, T.S. (2003) Symptoms experience: A concept analysis. Oncology Nursing Forum, 30, 601-606. http://dx.doi.org/10.1188/03.ONF.601-606

[27] Petrie, K.J. and Weinman, J. (2006) Why illness perception matters. Clinical Medicine, 6, 536-539. http://dx.doi.org/10.7861/clinmedicine.6-6-536

[28] Posey, A.D. (2006) Symptom perception: A concept exploration. Nursing Forum, 41, 113-124. http://dx.doi.org/10.1111/j.1744-6198.2006.00047.x

[29] Kleinman, A. (1988) The illness narratives: Suffering, healing, and the human condition. Basic Books, New York.

[30] Frank A.W. (1995) The wounded storyteller. Body, illness, and ethics. Chicago Press, Chicago. http://dx.doi.org/10.7208/chicago/9780226260037.001.0001

[31] Rosenman, S. (2008) Metaphor in clinical practice. Australian Family Physician, 37, 865-866.

[32] Eldh, A.-C., Ekman, I. and Ehnfors, M. (2006) Conditions for patient participation and non-participation in health care. Nursing Ethics, 13, 503-514. http://dx.doi.org/10.1191/0969733006nej898oa

[33] Ekman, I., Wolf, A., Olsson, L.E., Taft, C., Dudas, K., Schaufelberger, M. and Swedberg, K. (2012) Effects of person-centred care in patients with chronic heart failure: The PCC-HF study. European Heart Journal, 33, 11121119. http://dx.doi.org/10.1093/eurheartj/ehr306

[34] Ursel, K.L. and Aquino-Russell, C.E. (2010) Illuminating person-centered care with parse's teaching-learning model. Nursing Science Quarterly, 23, 118-123. http://dx.doi.org/10.1177/0894318410362546

[35] Edvardsson, D., Fetherstonhaugh, D., McAuliffe, L., Nay, R. and Chenco, C. (2011) Job satisfaction amongst aged care staff: Exploring the influence of person-centered care provision. International Psychogeriatrics, 23, 12051212. http://dx.doi.org/10.1017/S1041610211000159

[36] Skott, C. (2008) Symptoms beyond diagnosis-A case study. European Journal of Cancer Care, 17, 549-556.

[37] Corwin, E.J., Meek P., Cook P.F., Lowe N.K. and Sousa, K.H. (2012) Shape shifters: Biobehavioral determinants and phenomena in symptom research. Nursing Outlook, 60, 191-197. http://dx.doi.org/10.1016/j.outlook.2012.04.008

[38] Rosemond, C.A., Hanson, L.C., Ennett, S.T., Schenck, A.P. and Weiner, B.J. (2012) Implementing person-centered care in nursing homes. Health Care Management Review, 37, 257-266. http://dx.doi.org/10.1097/HMR.0b013e318235ed17

[39] Mezzich, J., Salloum, I., Cloninger, C., Salvador-Carulla, L., Kirmayer, L., Banzato, C., Wallcraft, J. and Botbol, M. (2010) Person-centred integrative diagnosis: Conceptual bases and structural model. Canadian Journal of Psychiatry, 55, 701-708.

[40] Ekman, I., Swedberg, K., Taft, C., Lindseth, A., Norberg, A., Brink, E., Carlsson, J., Dahlin-Ivanoff, S., Johansson, I.L., Kjellgren, K., Lidén, E., Öhlén, J., Olsson, L.E., Rosén, H., Rydmark, M. and Sunnerhagen, K.S. (2011) Person-centered care-Ready for prime time. European Journal of Cardiovascular Nursing, 10, 248-251. http://dx.doi.org/10.1016/j.ejcnurse.2011.06.008

[41] Kirkley, C., Bamford, C., Poole, M., Arksey, H., Hughes, J. and Bond, J. (2011) The impact of organisational culture on the delivery of person-centred care in services providing respite care and short breaks for people with dementia. Health and Social Care, 19, 438-448. http://dx.doi.org/10.1111/j.1365-2524.2011.00998.x

[42] McCormack, B., Dewing, J. and McCance, T. (2011) Developing person-centred care: Addressing contextual challenges through practice development. Online Journal of Issues in Nursing, 16, 3. 